\title{
The impact of levothyroxine on thyroid autoimmunity and hypothalamic-pituitary-thyroid axis activity in men with autoimmune hypothyroidism and early-onset androgenetic alopecia
}

\author{
Robert Krysiak ${ }^{(1)}$, Karolina Kowalcze², Bogdan Marek®33,4, Bogusław Okopieńn \\ ${ }^{1}$ Department of Internal Medicine and Clinical Pharmacology, Medical University of Silesia, Katowice, Poland \\ ${ }^{2}$ Department of Paediatrics in Bytom, School of Health Sciences in Katowice, Medical University of Silesia, Katowice, Poland \\ ${ }^{3}$ Division of Pathophysiology, Department of Pathophysiology and Endocrinology, Medical University of Silesia, Zabrze, Poland \\ ${ }^{4}$ Department of Endocrinology and Metabolic Diseases, Regional Specialist Hospital No. 3, Rybnik, Poland
}

\begin{abstract}
Introduction: Administration of testosterone or dehydroepiandrosterone to subjects with low levels of these hormones was found to reduce thyroid antibody titres. Male-pattern baldness is accompanied by mildly increased androgen levels. The present study was aimed at investigating whether early-onset androgenetic alopecia determines the impact of exogenous levothyroxine on thyroid autoimmunity and hypothalamic-pituitary-thyroid axis activity in young men with autoimmune hypothyroidism.

Material and methods: The study included 2 thyroid-antibody-matched groups of men with autoimmune hypothyroidism: subjects with early-onset androgenetic alopecia (group $1 ; n=24$ ) and subjects with no evidence of hair loss (group 2; $\mathrm{n}=24$ ). All patients were treated with exogenous levothyroxine. Circulating titres of thyroid peroxidase and thyroglobulin antibodies, as well as levels of thyrotropin, free thyroxine, free triiodothyronine, prolactin, total testosterone, calculated bioavailable testosterone, dehydroepiandrosterone-sulphate, and oestradiol were measured before levothyroxine treatment and 6 months later.

Results: In both study groups, levothyroxine decreased thyroid antibody titres, reduced thyrotropin levels and increased free thyroid hormone levels. However, these effects were less pronounced in the men with early-onset male-pattern baldness than in the control men. The degree of reduction in antibody titres and thyrotropin levels correlated with baseline levels of total and calculated bioavailable testosterone, as well with baseline insulin sensitivity and treatment-induced improvement in insulin sensitivity. Concentrations of the remaining variables remained unchanged throughout the study period.

Conclusions: The results of the current study suggest that the benefits of levothyroxine therapy in men with autoimmune hypothyroidism are less pronounced in individuals with early-onset androgenetic alopecia. (Endokrynol Pol 2021; 72 (5): 498-504)
\end{abstract}

Key words: androgenetic alopecia; hormones; hypothalamic-pituitary-thyroid axis; levothyroxine; thyroid autoimmunity

\section{Introduction}

A more frequent prevalence of autoimmune thyroid disease in women than in men may result from differences in sex hormone secretion [1]. There are some arguments suggesting that physiological concentrations of androgens minimize the risk of thyroid autoimmunity [2]. Subjects with Klinefelter's syndrome, the most common cause of male hypogonadism, are at increased risk of autoimmune hypothyroidism [3]. Men with autoimmune thyroid disease were characterized by lower values of the oestradiol:testosterone ratio than men without thyroid antibodies [4]. Shorter AR (CAG)n repeats of the androgen receptor gene, associated with its increased transcriptional activity, correlated with a younger age of onset of Hashimoto's thyroiditis [5]. Finally, exogenous androgens administered to men with their deficiency reduced thyroid autoimmunity and improved activity of the hypothalamic-pituitary-thyroid axis in men. The decrease in thyroid antibody titres, which was paralleled by the increase in thyroid's secretory capacity, was observed in testosterone-treated men with autoimmune thyroiditis coexisting with hypotestosteronaemia [6], as well as in DHEA-treated subjects with mild autoimmune thyroid hypofunction and low dehydroepiandrosterone-sulphate (DHEA-S) levels [7]. Testosterone replacement therapy also potentiated the impact of vitamin D on thyroid autoimmunity and thyroid function [8]. Because of relatively low post-treatment androgen levels, these findings do 
not allow us to conclude whether elevated concentrations of androgens affect thyroid autoimmunity and thyroid function.

Androgenetic alopecia, commonly known as male-pattern baldness, is the most common type of progressive hair loss disorder in men, affecting as much $30 \%$ of individuals by the age of 30 years and $80 \%$ of men during their lifespan $[9,10]$. Hair loss typically involves the temporal and vertex regions and mid-frontal scalp while sparing the occipital region [10]. Androgenetic alopecia diagnosed before the age of 30-35 years often occurs in first-generation relatives of women with polycystic ovary syndrome and is commonly regarded as the phenotypic equivalent of polycystic ovary syndrome in men, which carries the risk of developing obesity, metabolic syndrome, type 2 diabetes, and cardiovascular disease [11]. It seems that early-onset androgenetic alopecia is a state of mild androgen excess. Compared with subjects with no evidence of hair loss, men with early-onset male-pattern baldness are characterized by elevated levels of testosterone and dehydroepiandrosterone-sulphate (DHEA-S), lower levels of sex hormone-binding globulin, and higher values of the free androgen index [12]. The purpose of this study was to assess whether the presence of early-onset androgenetic alopecia in men determines the impact of exogenous levothyroxine on thyroid autoimmunity and hypothalamic-pituitary-thyroid activity.

\section{Material and methods}

The study protocol was approved by the Institutional Review Board and conducted in accordance with the Declaration of Helsinki. All included patients provided written informed consent after receiving an explanation of the voluntary nature of their participation and a description of the study.

\section{Patients}

The study population was selected from young men (18-40 years old) with untreated autoimmune hypothyroidism. Individuals were considered eligible for enrolment if they had the following: (a) thyroid peroxidase antibody (TPOAb) titres above $100 \mathrm{U} / \mathrm{mL}$; (b) sonographic features of Hashimoto's thyroiditis (diffuse hypoechoic echogenicity, parenchymal heterogeneity, hypoechoic micronodularity, echogenic septations, and hypervascularity); (c) serum thyrotropin levels in the range between 4.5 and $10.0 \mathrm{mU} / \mathrm{L}$ (d) free thyroxine levels in the range between 10.1 and $21.2 \mathrm{pmol} / \mathrm{L}$; and (d) free triiodothyronine in the range between 2.3 and 6.5 $\mathrm{pmol} / \mathrm{L}$. The study population was divided into two groups, each of which consisted of 24 patients. Power calculation showed that 21 subjects per group were needed to detect a $20 \%$ difference in thyroid antibody titres between both groups, with $80 \%$ power and a significance level of 0.05 . Three extra subjects per group were recruited in order to compensate for possible withdrawals. Group 1 included men with early-onset androgenetic alopecia, defined as grade 3 vertex or more alopecia on the Hamilton and Norwood scale (used to classify the stages of male pattern baldness $[13,14]$ ) by the age of 30 years. In turn, group 2 , serving as a control group, included subjects with no evidence of hair loss. Both groups of patients were selected among a larger group of men with autoimmune thyroid hypofunction ( $\mathrm{n}=162)$, using a computer program, with the aim of obtaining two study populations, matched for age, body mass index, blood pressure, thyroid antibody titres, and thyrotropin levels. In order to limit the effect of seasonal fluctuations in the outcome variables approximately half of the participants $(n=25)$ were enrolled in August or September and the remaining 23 were enrolled in February or March.

The exclusion criteria were as follows: positive serum antibodies against thyrotropin receptor, other autoimmune or endocrine disorders, impaired renal or hepatic function, malabsorption syndrome, body mass index above $35 \mathrm{~kg} / \mathrm{m}^{2}$, any other serious disorder, any pharmacotherapy, and poor compliance with the study protocol.

\section{Study design}

Over the entire study period, the participants were treated with exogenous levothyroxine. To avoid erratic absorption of levothyroxine, patients were instructed to take this drug on an empty stomach 30-60 minutes before breakfast (between 7.30 and 8.30 a.m.). The drug was administered at a starting daily dose of $37.5 \mu \mathrm{g}$. After two weeks, this dose was titrated to the final daily dose $(75 \mu \mathrm{g})$, which was administered for the following 6 months. Medication adherence was assessed every two months by means of a four-item Morisky-Green test, and the pill count and was regarded as satisfactory if all four questions in the Polish version of the Morisky-Green test were correctly answered and the percentage of tablets returned ranged between $0 \%$ and $10 \%$.

\section{Laboratory assays}

All measurements were carried out in duplicate (to ensure the consistency of assessments) at the beginning and at the end of the study. Venous blood was drawn between 7.00 and 8.00 a.m. to avoid circadian variations in the assessed variables, after an overnight $12-\mathrm{h}$ fasting period. Plasma glucose and creatinine levels were measured using standard enzymatic methods (Roche Diagnostics, Basel Switzerland). Serum titres of TPOAb and thyroglobulin antibodies (TgAb), as well as serum levels of thyrotropin, free thyroxine, free triiodothyronine, DHEA-S, total testosterone, oestradiol, sexhormone binding globulin, prolactin, and insulin, were assayed by direct chemiluminescence using acridinium ester technology (ADVIA Centaur XP Immunoassay System, Siemens Healthcare Diagnostics, Munich, Germany). Bioavailable testosterone was estimated from total testosterone and sex hormone-binding globulin with the Vermeulen formula, using the online calculator (www. issam.ch/freetesto.htm). The homeostasis model assessment 1 of insulin resistance index (HOMA1-IR) was measured by multiplying fasting glucose $[\mathrm{mg} / \mathrm{dL}]$ and insulin $[\mathrm{mU} / \mathrm{L}]$ divided by 405 . The estimated glomerular filtration rate was calculated using the Modification of Diet in Renal Disease study equation.

\section{Statistical analysis}

All outcome variables were log-transformed to approximate a normal distribution. Both groups, including changes from baseline after adjustment for baseline values, were compared by Student's $t$-tests for independent samples. Student's paired $t$-test was used to identify differences between the means of variables in each study group. The $\chi^{2}$ test was employed to compare the proportional data. Correlations were assessed using Pearson's correlation coefficient (r). The level of significance was defined as a $\mathrm{p}$ value corrected for multiple testing below 0.05 .

\section{Results}

At entry, there were no significant between-group differences in age, smoking habits, body mass index, blood pressure, TPOAb and TgAb antibody titres, serum levels of thyrotropin, free thyroid hormones, prolactin and 
Table 1. Baseline characteristics of patients

\begin{tabular}{lccc}
\hline Variable & Group 1 $^{\text {a }}$ & Group 2 $^{\text {b }}$ & $\begin{array}{c}\text { p value } \\
\text { [Group 1 vs. Group 2] }\end{array}$ \\
\hline Number [n] & 24 & 24 & - \\
\hline Age [years; mean (SD)] & $31(6)$ & $30(6)$ & 0.5565 \\
\hline Smokers [\%] & 29 & 25 & - \\
\hline Body mass index [kg/m² mean (SD)] & $27.3(5.5)$ & $26.7(4.8)$ & 0.6891 \\
\hline Systolic blood pressure [mmHg; mean (SD)] & $121(12)$ & $119(11)$ & 0.5502 \\
\hline Diastolic blood pressure [mmHg; mean (SD)] & $85(4)$ & $84(4)$ & 0.3910 \\
\hline
\end{tabular}

${ }^{a}$ men with early-onset androgenetic alopecia; ${ }^{b}$ men with normal hair growth; SD — standard deviation

oestradiol, as well as in the glomerular filtration rate (Tab. 1 and 2). Concentrations of DHEA-S total testosterone and calculated bioavailable testosterone, as well as values of HOMA1-IR, were higher in group 1 than in group 2. No significant adverse effects or any other complications were reported over the entire study period, and all participants completed the study protocol.
In both study groups, levothyroxine decreased the $\mathrm{TPOAb}$ and $\mathrm{TgAb}$ titres, reduced thyrotropin levels and HOMA1-IR, and increased free thyroxine and free triiodothyronine levels. All these effects were stronger in group B than in group A. Concentrations of prolactin, DHEA-S, total testosterone, calculated bioavailable testosterone, and oestradiol, as well as

Table 2. The effect of levothyroxine on thyroid antibody titres, hormones, insulin sensitivity, and glomerular filtration rate in men with or without early-onset androgenetic alopecia and autoimmune hypothyroidism

\begin{tabular}{|c|c|c|c|}
\hline Variable & Group 1a & Group $2^{b}$ & $\begin{array}{c}\text { p value } \\
\text { [Group } 1 \text { vs. Group 2] }\end{array}$ \\
\hline \multicolumn{4}{|l|}{ TPOAb [IU/mL; mean (SD)] } \\
\hline At the beginning of the study & $920(301)$ & $897(315)$ & 0.7971 \\
\hline At the end of the study & $723(256)$ & $565(227)^{*}$ & 0.0284 \\
\hline $\mathrm{p}$ value [post-treatment $v s$. baseline] & 0.0185 & 0.0001 & - \\
\hline \multicolumn{4}{|l|}{$\operatorname{TgAb}[\mathrm{IU} / \mathrm{mL} ;$ mean (SD)] } \\
\hline At the beginning of the study & $846(358)$ & $874(242)$ & 0.7523 \\
\hline At the end of the study & $658(243)$ & $521(219)^{*}$ & 0.0459 \\
\hline $\mathrm{p}$ value [post-treatment $v s$. baseline] & 0.0387 & $<0.0001$ & - \\
\hline \multicolumn{4}{|l|}{ Thyrotropin [mlU/L; mean (SD)] } \\
\hline At the beginning of the study & $7.3(1.5)$ & $7.4(1.4)$ & 0.8124 \\
\hline At the end of the study & $4.6(2.0)$ & $2.8(1.0)^{*}$ & 0.0003 \\
\hline $\mathrm{p}$ value [post-treatment $v s$. baseline] & $<0.0001$ & $<0.0001$ & - \\
\hline \multicolumn{4}{|l|}{ Free thyroxine [pmol/L; mean (SD)] } \\
\hline At the beginning of the study & $13.9(2.5)$ & $14.2(2.3)$ & 0.6673 \\
\hline At the end of the study & $17.2(2.9)$ & $19.0(3.0)^{*}$ & 0.0400 \\
\hline $\mathrm{p}$ value [post-treatment $v s$. baseline] & 0.0001 & $<0.0001$ & - \\
\hline \multicolumn{4}{|c|}{ Free triiodothyronine [pmol/L; mean (SD)] } \\
\hline At the beginning of the study & $3.2(0.6)$ & $3.2(0.5)$ & 1.0000 \\
\hline At the end of the study & $3.6(0.7)$ & $4.1(0.8)^{*}$ & 0.0258 \\
\hline$p$ value [post-treatment $v s$. baseline] & 0.0389 & $<0.0001$ & - \\
\hline \multicolumn{4}{|l|}{ Prolactin [ng/mL; mean (SD) } \\
\hline At the beginning of the study & $13.1(5.1)$ & $11.9(5.2)$ & 0.4237 \\
\hline At the end of the study & $12.7(5.8)$ & $10.0(4.4)$ & 0.0758 \\
\hline $\mathrm{p}$ value [post-treatment vs. baseline] & 0.8008 & 0.1784 & - \\
\hline
\end{tabular}


Table 2. The effect of levothyroxine on thyroid antibody titres, hormones, insulin sensitivity, and glomerular filtration rate in men with or without early-onset androgenetic alopecia and autoimmune hypothyroidism

\begin{tabular}{|c|c|c|c|}
\hline Variable & Group 1a & Group $2^{b}$ & $\begin{array}{c}\text { p value } \\
\text { [Group } 1 \text { vs. Group 2] }\end{array}$ \\
\hline \multicolumn{4}{|l|}{ DHEA-S [ $\mu \mathrm{mol} / \mathrm{L} ;$ mean (SD)] } \\
\hline At the beginning of the study & $7.8(3.0)$ & $5.3(2.0)$ & 0.0014 \\
\hline At the end of the study & $7.5(1.9)$ & $5.1(2.1)$ & 0.0001 \\
\hline $\mathrm{p}$ value [post-treatment $v s$. baseline] & 0.6809 & 0.7370 & - \\
\hline \multicolumn{4}{|l|}{ Total testosterone [nmol/L; mean (SD)] } \\
\hline At the beginning of the study & $27.0(8.2)$ & $21.1(6.4)$ & 0.0079 \\
\hline At the end of the study & $26.5(7.1)$ & $20.4(5.8)$ & 0.0021 \\
\hline $\mathrm{p}$ value [post-treatment $v s$. baseline] & 0.8223 & 0.6932 & - \\
\hline \multicolumn{4}{|c|}{ Calculated free testosterone [pmol/L; mean (SD)] } \\
\hline At the beginning of the study & $12.2(3.2)$ & $7.9(2.5)$ & $<0.0001$ \\
\hline At the end of the study & $11.4(3.4)$ & $7.4(2.1)$ & $<0.0001$ \\
\hline $\mathrm{p}$ value [post-treatment $v s$. baseline] & 0.4056 & 0.4569 & - \\
\hline \multicolumn{4}{|l|}{ Oestradiol [pmol/L; mean (SD)] } \\
\hline At the beginning of the study & $153(48)$ & $146(40)$ & 0.5858 \\
\hline At the end of the study & $149(39)$ & $135(42)$ & 0.2376 \\
\hline $\mathrm{p}$ value [post-treatment $v s$. baseline] & 0.7528 & 0.3577 & - \\
\hline \multicolumn{4}{|l|}{ HOMA1-IR [mean (SD)] } \\
\hline At the beginning of the study & $3.4(1.2)$ & $2.8(0.8)$ & 0.0473 \\
\hline At the end of the study & $2.6(1.0)$ & $1.5(0.7)^{*}$ & 0.0001 \\
\hline $\mathrm{p}$ value [post-treatment $v s$. baseline] & 0.0157 & $<0.0001$ & - \\
\hline $\begin{array}{l}\text { Estimated glomerular filtration rate } \\
{\left[\mathrm{mL} / \mathrm{min} / 1.73 \mathrm{~m}^{2} ; \text { mean }(\mathrm{SD})\right]}\end{array}$ & & & - \\
\hline At the beginning of the study & $92(20)$ & $89(15)$ & 0.5595 \\
\hline At the end of the study & $98(18)$ & $96(22)$ & 0.7319 \\
\hline $\mathrm{p}$ value [post-treatment $v s$. baseline] & 0.2803 & 0.2042 & - \\
\hline
\end{tabular}

${ }^{a}$ men with early-onset androgenetic alopecia; ${ }^{b}$ men with normal hair growth; Statistically significant results are marked in bold; *the impact of levothyroxine [percent changes from baseline after adjustment for baseline values] stronger than in group $\mathrm{A}$

the glomerular filtration rate, remained unchanged throughout the study period. Both groups differed in post-treatment values of antibody titres, post-treatment levels of thyrotropin, free thyroid hormones, DHEA$S$ total testosterone, bioavailable testosterone, and in HOMA1-IR (Tab. 2).

At entry, antibody titres correlated with thyrotropin levels [TPOAb: $\mathrm{r}=0.51$ ( $\mathrm{p}<0.0001)$, TgAb: $r=0.43(p=0.0005)]$, total testosterone [TPOAb: $r=0.31(p=0.0221), \operatorname{TgAb}: r=0.26(p=0.0355)]$, and calculated free testosterone [TPOAb: $r=0.35$ $(p=0.0056), \operatorname{TgAb}: r=0.32(p=0.0041)]$. TPOAb titres correlated also with DHEA-S $[\mathrm{r}=0.28$ ( $\mathrm{p}=0.0392)]$, while androgen levels correlated with HOMA1-IR [total testosterone: $\mathrm{r}=0.32(\mathrm{p}=0.0195)$; bioavailable testosterone: $r=0.41$ ( $p=0.0007)$; DHEA-S: $r=0.25$ $(p=0.0458)]$. Treatment-induced changes in antibody titres correlated with baseline antibody titres [group
$1-$ TPOAb: $r=0.34(p=0.0098)$, TgAb: $r=0.28$ $(\mathrm{p}=0.0402)$; group $2-$ TPOAb: $\mathrm{r}=0.37(\mathrm{p}=0.0084)$ ， TgAb: $r=0.31(p=0.0187)$ ], total testosterone [group $1-$ TPOAb: $r=-0.39(p=0.0012]$, TgAb: $r=-0.35$ $(\mathrm{p}=0.0088)$; group $2-$ TPOAb: $r=0.47(\mathrm{p}=0.0002)$, TgAb: $r=0.25(p=0.0488)]$, and calculated bioavailable testosterone [group $1-$ TPOAb: $\mathrm{r}=0.43(\mathrm{p}=0.0008)$, TgAb: $r=-0.37(p=0.0069)$; group $2-$ TPOAb: $r=0.44$ $(p=0.0003), \operatorname{TgAb}: r=0.29(p=0.0274)]$. There were also correlations between treatment-induced reduction in antibody titres and the effect of treatment on thyrotropin levels [group $1-$ TPOAb: $r=0.55(p<0.0001)$, TgAb: $r=0.48(p=0.0001)$; group $2-$ TPOAb: $r=0.37$ $(p<0.0075)$, TgAb: $r=0.29(p=0.0283)]$ and on HOMA1-IR [group $1-$ TPOAb: $\mathrm{r}=0.40(\mathrm{p}=0.0010)$, TgAb: $\mathrm{r}=0.28(\mathrm{p}=0.0387)$; group $2-$ TPOAb: $\mathrm{r}=0.38$ $(p=0.0040)$, TgAb: $r=0.24(p=0.0495)]$. No other correlations were significant. 


\section{Discussion}

Despite the selection procedure used in the current study, the subjects with early-onset androgenetic alopecia had elevated levels of total and calculated testosterone and of DHEA-S, which suggests that they are characterized by increased gonadal and adrenal steroidogenesis. However, the androgen profile of these individuals differed from those observed in male siblings of women with polycystic ovary syndrome, in whom elevated levels of DHEA-S coexisted with decreased values of calculated bioavailable testosterone, which suggested impaired conversion of dehydroepiandrosterone to androstenedione or androstenedione to testosterone [15]. This finding indicates that men with early-onset androgenetic alopecia are phenotypically distinct from brothers of women with polycystic ovary syndrome. The obtained results also indicate that subjects with early-onset male-pattern baldness are characterized by impaired insulin sensitivity, which may partially explain their increased susceptibility to the development of obesity, metabolic syndrome, and type 2 diabetes [11].

Another interesting observation resulting from our study is that men with early-onset androgenetic alopecia may be characterized by a more severe course of Hashimoto's thyroiditis than men with normal hair growth. Although the selection procedure was aimed at obtaining two study groups with similar titres of thyroid antibodies, TPOAb and TgAb titres correlated with both total and bioavailable testosterone levels. Moreover, titres of TPOAb, which are regarded as more sensitive and specific for thyroid autoimmune diseases than titres of TgAb [16], correlated with DHEA-S levels. Enhanced autoimmune destruction of the thyroid gland may be mediated by increased production of proinflammatory cytokines. In line with this explanation, anabolic steroids induced production of tumour necrosis- $\alpha$ and interleukin-1 $\beta$ [17] and their increased production by inflammatory cells correlated with severity of autoimmune thyroiditis [18].

However, the major finding of the present study is that men with autoimmune hypothyroidism benefit to a lesser extent from levothyroxine treatment if they have coexisting early-onset androgenetic alopecia. This observation seems to be clinically relevant because early-onset male-pattern baldness can be easily diagnosed based on anamnesis and clinical examination. The obtained results, as well as previous observations about increased cardiometabolic risk ${ }^{10}$, show that early-onset androgenetic alopecia cannot be regarded as only a cosmetic defect but also a real danger to health. In light of previously described effects of androgen therapy on thyroid autoimmunity and on the results of thyroid function tests [6-8], it seems that there exists an inverted U-shaped relationship between the impact of pharmacotherapy on thyroid autoimmunity and androgen levels. Therefore, both androgen deficiency and androgen excess may induce or deteriorate the course of Hashimoto's thyroiditis.

The study design does not provide a mechanistic explanation for the obtained results. The most plausible explanation is a direct modulatory effect of endogenous androgens. In line with this hypothesis, the impact on antibody titres correlated with circulating levels of all measured androgens. The finding that this correlation was strongest for calculated bioavailable testosterone probably results from the fact that bioavailable testosterone is the biologically active fraction of testosterone that is not tightly bound to sex hormone-binding globulin, and more accurately than total testosterone reflects the clinical androgen state of men $[19,20]$. Moreover, if calculated by Vermeulen's formula, bioavailable testosterone levels highly correlate with free testosterone levels measured by equilibrium dialysis [20].

According to the second explanation, a weaker effect of levothyroxine treatment may be secondary to the impaired insulin sensitivity observed in the participants of the present study. In line with this hypothesis, the degree of reduction in thyroid antibody titres correlated with both baseline and treatment-induced changes in HOMA1-IR. Moreover, Liu et al. [21] reported that TPOAb titres correlated with HOMA1-IR and the concentration of C-reactive protein in non-obese individuals with normal thyroid function, suggesting that mild deviation of thyroid function within the normal range, chronic inflammation, and insulin resistance may be a link between thyroid autoimmunity and metabolic abnormalities in the non-obese population. The presence of correlations between total and bioavailable testosterone levels and HOMA1-IR may indicate that elevated androgen levels increase insulin resistance, which is in line with previous findings of our research team [22]. If this explanation is correct, men with autoimmune hypothyroidism may be candidates for combined administration of levothyroxine with metformin. Interestingly, metformin administered to subjects with primary thyroid hypofunction reduces elevated thyrotropin levels [23], and by modulating thyrotrope function it may potentiate the impact of levothyroxine on hypothalamic-pituitary-thyroid axis activity.

The association between early-onset androgenetic alopecia and levothyroxine action on thyroid autoimmunity and hypothalamic-pituitary-thyroid axis activity is unlikely to be mediated by oestrogens or prolactin. The conversion of testosterone to oestradiol catalysed by aromatase may in subjects with early-onset androgenetic alopecia theoretically result in mild oestrogen 
excess. However, this explanation is not supported by the results of the current study. There were no differences in oestradiol levels (which were within the reference range), levothyroxine produced a neutral effect on oestradiol concentrations, while oestradiol levels did not correlate with levothyroxine-induced changes in antibody titres or hormone levels. The obtained results cannot be also attributed to prolactin excess, reported in up to $22 \%$ of patients with subclinical thyroid hypofunction [24] and more frequently observed in men with adult-onset androgenetic alopecia [12]. Although this hormone exerts a complex proinflammatory effect (impairing the negative selection of autoreactive B lymphocytes during B cell maturation, stimulating the maturation of $\mathrm{T}$ cells, inhibiting apoptosis of lymphocytes, and enhancing the proliferative response to antigens and mitogens [25,26]), its levels in the current study remained unchanged and did not correlate with antibody titres and hypothalamic-pituitary-thyroid axis activity.

Our study has several limitations that should be considered when interpreting our findings. Although it exceeded the required sample size, the study included a relatively small number of participants, and the obtained results need to be confirmed in a large prospective trial. The study design did not allow us to totally eliminate the "regression toward the mean" effect, arising when an extreme variable on the first measurement is closer to the average on subsequent measurements [27]. Furthermore, the study population was characterized by sufficient iodine [28] and low selenium $[29,30]$ intake. It is not certain whether the impact of levothyroxine treatment is similar in patients inhabiting iodine-deficient and selenium-sufficient areas. Finally, it cannot be totally excluded that the effect of early-onset androgenetic alopecia on levothyroxine action is different in euthyroid subjects with autoimmune thyroiditis and in subjects with overt autoimmune hypothyroidism, not participating in the present study.

\section{Conclusions}

Although levothyroxine treatment reduced thyroid antibody titres and altered thyrotropin and thyroid hormone levels in both study groups, these effects were more pronounced in men with normal hair growth than in men with early-onset androgenetic alopecia. The degree of reduction in antibody titres depended on baseline androgen levels, as well as on insulin sensitivity and treatment-induced improvement in insulin sensitivity. The obtained results suggest that men with early-onset androgenetic alopecia benefit to a lesser degree from levothyroxine therapy than the remaining men with autoimmune thyroid hypofunction.

\section{Acknowledgements}

This research received no specific grant from any funding agency in the public, commercial, or not-for-profit sectors.

\section{Declaration of interest statement}

The authors declare no financial interests.

\section{References}

1. Gessl A, Lemmens-Gruber R, Kautzky-Willer A. Thyroid disorders. Handb Exp Pharmacol. 2012(214): 361-386, doi: 10.1007/978-3-64 2-30726-3 17, indexed in Pubmed: 23027459.

2. Bajuk Studen K, Biček A, Oblak A, et al. Hypothyroidism is associated with higher testosterone levels in postmenopausal women with Hashimoto's thyroiditis. Endokrynol Pol. 2020; 71(1): 73-75, doi: 10.5603/EP.a2019.0055, indexed in Pubmed: 31681972.

3. Rao A, Jain D, Aggarwal HK, et al. An enigmatic trio of Klinefelter's syndrome, autoimmune hypothyroidism and nephrotic syndrome. J R Coll Physicians Edinb. 2017; 47(2): 143-145, doi: 10.4997/JRCPE.2017.208, indexed in Pubmed: 28675185.

4. Chen Yi, Chen Y, Xia F, et al. A Higher Ratio of Estradiol to Testosterone Is Associated with Autoimmune Thyroid Disease in Males. Thyroid. 2017; 27(7): 960-966, doi: 10.1089/thy.2016.0661, indexed in Pubmed: 28558486

5. Doukas C, Saltiki K, Mantzou A, et al. Hormonal parameters and sex hormone receptor gene polymorphisms in men with autoimmune diseases. Rheumatol Int. 2013; 33(3): 575-582, doi: 10.1007/s00296-012-2386-4, indexed in Pubmed: 22466402

6. Krysiak R, Kowalcze K, Okopien B. The effect of testosterone on thyroid autoimmunity in euthyroid men with Hashimoto's thyroiditis and low testosterone levels. J Clin Pharm Ther. 2019; 44(5): 742-749, doi: 10.1111/jcpt.12987, indexed in Pubmed: 31183891.

7. Krysiak R, Szkróbka W, Okopień B. Impact of dehydroepiandrosterone on thyroid autoimmunity and function in men with autoimmune hypothyroidism. Int J Clin Pharm. 2020 [Epub ahead of print], doi: 10.1007/s11096-020-01207-w, indexed in Pubmed: 33245519.

8. Krysiak R, Kowalcze K, Okopień B. The effect of vitamin D on thyroid autoimmunity in euthyroid men with autoimmune thyroiditis and testosterone deficiency. Pharmacol Rep. 2019; 71(5): 798-803, doi: 10.1016/j. pharep.2019.04.010, indexed in Pubmed: 31377561.

9. Kelly Y, Blanco A, Tosti A. Androgenetic Alopecia: An Update of Treatment Options. Drugs. 2016; 76(14): 1349-1364, doi: 10.1007/s40265-016-0629-5, indexed in Pubmed: 27554257.

10. Lolli F, Pallotti F, Rossi A, et al. Androgenetic alopecia: a review. Endocrine. 2017; 57(1): 9-17, doi: 10.1007/s12020-017-1280-y, indexed in Pubmed: 28349362.

11. Cannarella R, Condorelli RA, Mongiò̀ LM, et al. Does a male polycystic ovarian syndrome equivalent exist? J Endocrinol Invest. 2018; 41(1): 49-57, doi: 10.1007/s40618-017-0728-5, indexed in Pubmed: 28711970.

12. Sanke S, Chander R, Jain A, et al. A Comparison of the Hormonal Profile of Early Androgenetic Alopecia in Men With the Phenotypic Equivalent of Polycystic Ovarian Syndrome in Women. JAMA Dermatol. 2016; 152(9): 986-991, doi: 10.1001/jamadermatol.2016.1776, indexed in Pubmed: 27304785

13. Hamilton JB. Patterned loss of hair in man; types and incidence. Ann N Y Acad Sci. 1951; 53(3): 708-728, doi: 10.1111/j.1749-6632.1951.tb31971.x, indexed in Pubmed: 14819896.

14. Norwood OT. Male pattern baldness: classification and incidence. South Med J. 1975; 68(11): 1359-1365, doi: 10.1097/00007611-197511000-00009, indexed in Pubmed: 1188424.

15. Krysiak R, Szkróbka W, Okopień B. The impact of atorvastatin on cardiometabolic risk factors in brothers of women with polycystic ovary syndrome. Pharmacol Rep. 2021; 73(1): 261-268, doi: 10.1007/s43440-020-00135-w, indexed in Pubmed: 32696349.

16. Muller AF, Drexhage HA, Berghout A. Postpartum thyroiditis and autoimmune thyroiditis in women of childbearing age: recent insights and consequences for antenatal and postnatal care. Endocr Rev. 2001; 22(5): 605-630, doi: 10.1210/edrv.22.5.0441, indexed in Pubmed: 11588143

17. Hughes T, Fulep E, Juelich T, et al. Modulation of immune responses by anabolic androgenic steroids. Int J Immunopharmacol. 1995; 17(11): 857-863, doi: 10.1016/0192-0561(95)00078-x, indexed in Pubmed: 8788115

18. Krysiak R, Okopien B. The effect of levothyroxine and selenomethionine on lymphocyte and monocyte cytokine release in women with Hashimoto's thyroiditis. J Clin Endocrinol Metab. 2011; 96(7): 2206-2215, doi: 10.1210/jc.2010-2986, indexed in Pubmed: 21508145.

19. Keevil BG, Adaway Jo. Assessment of free testosterone concentration. J Steroid Biochem Mol Biol. 2019; 190: 207-211, doi: 10.1016/j. jsbmb.2019.04.008, indexed in Pubmed: 30970279. 
20. Ho CKM, Stoddart M, Walton M, et al. Calculated free testosterone in men: comparison of four equations and with free androgen index. Ann Clin Biochem. 2006; 43(Pt 5): 389-397, doi: 10.1258/000456306778520115, indexed in Pubmed: 17036414.

21. Liu J, Duan Y, Fu J, et al. Association Between Thyroid Hormones, Thyroid Antibodies, and Cardiometabolic Factors in Non-Obese Individuals With Normal Thyroid Function. Front Endocrinol (Lausanne). 2018; 9: 130, doi: 10.3389/fendo.2018.00130, indexed in Pubmed: 29674996.

22. Krysiak R, Kowalcze K, Marek B, et al. Cardiometabolic risk factors in women with non-classic congenital adrenal hyperplasia. Acta Cardiol. 2020; 75(8): 705-710, doi: 10.1080/00015385.2019.1666964, indexed in Pubmed: 31538536.

23. Meng X, Xu S, Chen G, et al. Metformin and thyroid disease. J Endocrinol. 2017; 233(1): R43-R51, doi: 10.1530/JOE-16-0450, indexed in Pubmed: 28196954.

24. Vilar L, Vilar CF, Lyra R, et al. Pitfalls in the Diagnostic Evaluation of Hyperprolactinemia. Neuroendocrinology. 2019; 109(1): 7-19, doi: 10.1159/000499694, indexed in Pubmed: 30889571.

25. De Bellis A, Bizzarro A, Pivonello R, et al. Prolactin and autoimmunity. Pituitary. 2005; 8(1): 25-30, doi: 10.1007/s11102-005-5082-5, indexed in Pubmed: 16411065 .
26. Shelly S, Boaz M, Orbach H. Prolactin and autoimmunity. Autoimmun Rev. 2012; 11(6-7): A465-A470, doi: 10.1016/j.autrev.2011.11.009, indexed in Pubmed: 22155203.

27. Barnett AG, van der Pols JC, Dobson AJ. Regression to the mean: what it is and how to deal with it. Int J Epidemiol. 2005; 34(1): 215-220, doi: 10.1093/ije/dyh299, indexed in Pubmed: 15333621.

28. Szybiński Z. Polish Council for Control of Iodine Deficiency Disorders. Work of the Polish Council for Control of Iodine Deficiency Disorders, and the model of iodine prophylaxis in Poland. Endokrynol Pol. 2012; 63(2): 156-160, indexed in Pubmed: 22538756.

29. Klapcinska B, Poprzecki S, Danch A, et al. Selenium Levels in Blood of Upper Silesian Population: Evidence of Suboptimal Selenium Status in a Significant Percentage of the Population. Biol Trace Elem Res. 2005; 108(1-3): 001-016, doi: 10.1385/bter:108:1-3:001, indexed in Pubmed: 16327055 .

30. Filipowicz D, Majewska K, Kalantarova A, et al. The rationale for selenium supplementation in patients with autoimmune thyroiditis, according to the current state of knowledge. Endokrynol Pol. 2021; 72(2): 153-162, doi: 10.5603/EP.a2021.0017, indexed in Pubmed: 33970480 . 